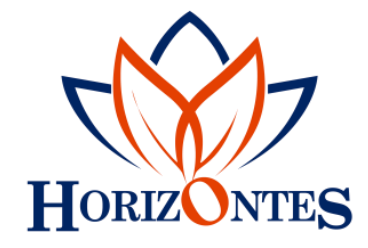

Horizontes. Revista de Investigación en Ciencias de la Educación Https://doi.org/10.33996/revistahorizontes.v5i17.159

Enero - marzo 2021

Volumen 5 / No. 17

ISSN: $2616-7964$

ISSN-L: 2616 - 7964

pp. $59-76$

www.revistahorizontes.org

\title{
Educación virtual en época de Covid-19: perspectiva de los educandos a nivel superior
}

\author{
Virtual education in the time of Covid-19: perspective of higher-level learners \\ Educação virtual na época da Covid-19: perspectiva dos alunos de nível superio
}

ARTÍCULO DE INVESTIGACIÓN

\section{Jorge Patricio Chávez Reinoso chavezj_ites@yahoo.es \\ ORCID: 0000-0003-1738-5181}

\author{
Víctor Manuel Flores Andino \\ victorfloresandino@hotmail.com \\ ORCID: 0000-0001-5686-6864
}

Jorge Ernesto Chávez Guevara z

jorgechavezguevara94@gmail.com jechavez@institutos.gob.ec

ORCID: 0000-0002-5627-4321

Ivonne Gabriela Guayanlema Chávez

gabriela_1806@hotmail.es

ORCID: 0000-0003-2794-5701

\section{Instituto Tecnológico Superior Carlos Cisneros, Ecuador}

Recibido 04 de febrero 2021 | Arbitrado y aceptado 16 de febrero 2021 | Publicado en marzo 2021

\section{RESUMEN}

El presente artículo tiene como objetivo conocer la perspectiva y comportamiento del alumnado acerca de la modalidad virtual en tiempo de pandemia por efecto de la suspensión de clases presenciales, producida por el Covid-19. El estudio fue descriptivo, transversal, de campo, documental y bibliográfico; con enfoque cuantitativo y cualitativo. Los resultados obtenidos indican que el $60 \%$ de los estudiantes estaban preparados desde el punto de vista educativo para enfrentar esta crisis, en cuanto a equipos tecnológicos se utilizaron en mayor porcentaje los computadores portátiles y de escritorio, la calidad del uso del internet estuvo entre bueno y regular, entre las actividades académicas realizadas resaltaron las clases virtuales, los trabajos grupales y el sistema teams fue el más usado para el dictado de clases. Se concluye que mediante la educación virtual no se lograron buenos resultados en cuanto al proceso de formación educativa, el nivel de enseñanza de los docentes y el aprendizaje de los estudiantes disminuyó comparando las etapas antes y durante la pandemia.

Palabras clave: Educación virtual; Covid-19; pandemia; educación superior; estudiantes, ESPOCH

\section{ABSTRACT}

The objective of this article is to know the perspective and behavior of students about the virtual modality in times of pandemic due to the suspension of face-to-face classes, produced by Covid-19. The study was descriptive, cross-sectional, field, documentary and bibliographic; with a quantitative and qualitative approach. The results obtained indicate that $60 \%$ of the students were prepared from an educational point of view to face this crisis, in terms of technological equipment, laptops and desktops were used in a higher percentage, the quality of Internet use was between good and regular, among the academic activities carried out, virtual classes stood out, group work and the teams system was the most used for teaching classes. It is concluded that through virtual education, good results were not achieved in terms of the educational training process, the level of teaching of teachers and student learning decreased when comparing the stages before and during the pandemic.

Key words: Virtual education; Covid-19; pandemic; higher education; students, ESPOCH 


\section{RESUMO}

O objetivo deste artigo é conhecer a perspectiva e o comportamento dos alunos sobre a modalidade virtual em tempos de pandemia devido à suspensão das aulas presenciais, produzida pela Covid-19. 0 estudo foi descritivo, transversal, de campo, documental e bibliográfico; com abordagem quantitativa e qualitativa. Os resultados obtidos indicam que $60 \%$ dos alunos estavam preparados do ponto de vista educacional para enfrentar esta crise, em termos de equipamentos tecnológicos, laptops e desktops foram utilizados em maior percentual, a qualidade de uso da Internet ficou entre boa e regular, dentre as atividades acadêmicas realizadas, destacaram-se as aulas virtuais, o trabalho em grupo e o sistema de equipes foi o mais utilizado para ministrar as aulas. Conclui-se que, por meio da educação virtual, não foram alcançados bons resultados em termos do processo de formação educacional, o nível de ensino dos professores e a aprendizagem dos alunos diminuíram na comparação das etapas pré e durante a pandemia.

Palavras-chave: Educação virtual; Covid19; pandemia; Educação superior; alunos, ESPOCH

\section{INTRODUCCIÓN}

La Organización Mundial de la Salud (OMS), en marzo del 2020 declaró en todo el mundo como pandemia la enfermedad de la Covit-19 o coronavirus, afectando a las diferentes actividades económicas, productivas, comerciales, sociales, turísticas y principalmente las educativas a nivel nacional e internacional, y con ello, las consecuencias que esta pandemia ha ocasionado.

De acuerdo con la Organización Mundial de la Salud -OMS- (2020):

Los coronavirus (CoV) son una amplia familia de virus que pueden causar diversas afecciones, desde el resfriado común hasta enfermedades más graves, como ocurre con el coronavirus causante del síndrome respiratorio de Oriente Medio (MERS-CoV) y el que ocasiona el síndrome respiratorio agudo severo (SRAS-CoV). Un nuevo coronavirus es una nueva cepa de coronavirus que no se había encontrado antes en el ser humano. (párr. 1).

El coronavirus (Covid-19), señalada por la Organización Mundial de la Salud (OMS), como una pandemia; representa una enfermedad debidamente comprobada, que ha generado mucho impacto a nivel mundial, en áreas productivas, social y económico, en la educación, en la salud, empleo, tecnología, entre otros.

De acuerdo con un estudio realizado por la UNESCO (2020):

Al 27 de abril de 2020, 35 países de la región habían decidido suspender las clases en todos los niveles educativos, lo que ha afectado a más de 115 millones de alumnos, desde el nivel preescolar hasta el terciario". Adicional, y sobre este mismo aspecto, "La interrupción de la educación ha tenido y seguirá teniendo efectos considerables en otros ámbitos además del educativo. Los cierres de instituciones educativas dificultan la prestación de servicios esenciales a niños $\mathrm{y}$ comunidades". (Naciones Unidas, 2020, p. 2).

Como consecuencia de aquello y en afán de precautelar la salud de los habitantes, el Gobierno del Ecuador decreto permanecer bajo cuarentena y aislamiento social en todo el territorio nacional, esta situación provocó que los diferentes sectores públicos y privados en diferentes áreas realicen sus actividades laborales a través de teletrabajo; por lo cual el sector educativo no estuvo exento de esta problemática, lo que generó que la educación en todos los niveles y especialmente el superior labore utilizando el sistema de educación virtual o en línea. 
Este artículo forma parte de un amplio estudio relacionado con la educación superior y la modalidad de enseñanza aprendizaje virtual, por ello Valladares y Poma (2015); indican que "la necesidad de la población de acceder a una educación superior de excelencia es algo latente en las sociedades del siglo XXI (...)” (p. 1). Igual, “La formación de jóvenes es un aspecto relevante y de impacto en el desarrollo global por lo cambios significativos que ellos pueden impulsar en la sociedad" (Reynoso, Pernas y Perazzo, 2017, p. 103). Además, Ceballos, Arévalo y Giraldo (2012), indican que "la educación constituye la escalera del conocimiento que permite avanzar tanto personal como profesionalmente, en esta medida es de gran importancia el crecimiento de una ciudad, un país y en general del mundo" (p. 31)

En este contexto es importante explorar también que: "El reto de los sistemas educativos en los últimos meses ha sido mantener la vitalidad de la educación y promover el desarrollo de aprendizaje significativos. Para ello, ha contado con dos aliados claves: sus docentes y la virtualidad, en términos más precisos, los docentes a través de la virtualidad". (Expósito y Marsollier, 2020, p. 2).

La educación superior en el mundo en general y en el Ecuador en particular, durante el tiempo de pandemia ha venido utilizando la modalidad de educación virtual, online o en línea, con todas sus ventajas y desventajas, aquí los docentes han tenido que reinventarse para poder llegar con varias estrategias educativas hacia sus estudiantes; al mismo tiempo los alumnos han entendido la importancia de esta modalidad, el rol y la responsabilidad que deben asumir en el proceso de enseñanza y aprendizaje.

Por ello, y como lo afirman Bravo-García y Magis-Rodríguez (2020), el estudio de la virtualidad cobra cada día más relevancia, debido a que la educación online en el 2020 es un hecho sin precedentes ya que marca un antes y un después en las prácticas pedagógicas yen todos los sistemas educativos actuales a nivel global, ya que se ha puesto en evidencia existen grandes desigualdades sociales, culturales y económicas en más de 180 países que han sido víctimas de la pandemia por COVID-19. (s/p).

Las diferentes investigaciones que existen hasta el momento, han demostrado que la educación virtual en la actualidad es el único mecanismo en los procesos de enseñanza y aprendizaje a nivel educativo y especialmente de la educación superior; por ello, se desarrolla un creciente interés por la educación en línea. Bajo este argumento la educación virtual representa una modalidad, que se refiere al desarrollo de programas de formación continua, que tiene como escenario en el proceso de enseñanza y aprendizaje el uso de la herramienta del internet o el ciberespacio; facilitando así la comunicación permanente entre los docentes tutores y el alumnado.

Estos argumentos, permiten analizar que la educación virtual representa la emergencia de un nuevo paradigma educativo, debido que este debe estar argumentado pedagógicamente bajo sustentos conceptuales y mapas mentales innovadores, a fin de establecer el encuentro comunicativo entre los actores del proceso. (Gutiérrez, 2016).

Concomitante con lo anotado en párrafos anteriores, estudios realizados por el Banco Interamericano de Desarrollo (BID, 2020), en relación con la educación virtual, afirman que la los lapsos y la velocidad de la modalidad de enseñanza remota está siendo implementada sin precedentes, por lo que se hace necesario la combinación de soluciones y medios de primera generación (materiales impresos, 
radio y televisión) y de segunda generación (plataformas, sistemas de gestión de aprendizajes) para poder cumplir el objetivo de educar a los estudiantes y que este aprendizaje llegue con la trasmisión de la enseñanza remota.

Por otro lado, en relación al tutor virtual, y de acuerdo con Velásquez (2020):

La tutoría virtual se construye a través de un proceso de acompañamiento en el aprendizaje, donde el éxito radica en una combinación de metodologías que fortalecen el desarrollo de la educación. No todos los docentes utilizan los entornos virtuales a pesar de que se tiene plataforma educativa y las herramientas que existen para el acompañamiento pedagógico, debido a la contingencia sanitaria que se vive actualmente se ven en la necesidad de aplicar la virtualidad, por lo tanto, se busca mediante la aplicación de teoría y conceptos básicos determinar $\mathrm{y}$ analizar las funciones y roles del tutor virtual, así como identificar las competencias idóneas que el tutor virtual debe poseer. (p. 20).

Adicionalmente, como la manifiesta en su estudio Marciniak y Gairín-Sallán (2018):

Para que una modalidad de educación virtual sea de calidad, debe contemplar ciertos requisitos, tales como: contar con los recursos tecnológicos adecuados y el servicio necesario para acceder al programa educativo; que la estructura y el contenido del curso virtual ofrezcan un valor formativo; que se realicen aprendizajes efectivos y que sea un ambiente satisfactorio tanto para los estudiantes como para los profesores.

En este contexto, el objetivo básico de esta investigación es conocer desde la perspectiva de los estudiantes de carreras empresariales de la Facultad de Administración de Empresas, en la Escuela Superior Politécnica de Chimborazo (ESPOCH), ciertas características del comportamiento de la educación virtual en tiempo de pandemia, por efecto de la suspensión de clases en la modalidad presencial, a consecuencia de la pandemia del coronavirus o Covit-19.

\section{METODOLOGÍA}

Estudio fue realizado durante la época de pandemia (Entre Junio y Julio del año 2020), siendo de carácter descriptivo, transversal, exploratorio y no experimental con enfoque mixto (cuantitativo y cualitativo), además se utilizó investigación de campo a través de la aplicación de encuestas; el artículo fue respaldado en la revisión bibliográfica y documental de libros digitales, revistas científicas, trabajos de titulación en universidades nacionales y extranjeras, páginas electrónicas, entre otros.

La población es "El conjunto de unidades de observación que poseen características específicas determinadas por el investigador según los objetivos que persigue" (Flores, 2011, p. 203). La población motivo de estudio estuvo conformada por el alumnado de carreras empresariales, de diferentes especialidades de la Facultad de Administración de Empresas, (ESPOCH), durante el periodo académico mayo-octubre 2020; que se presenta en la tabla 1. 
Tabla 1. Población y muestra del estudio

\begin{tabular}{ccc}
\hline CARRERAS & NUMERO DE ALUMNOS & $\begin{array}{c}\text { MUESTRA } \\
\text { DEFINITIVA }\end{array}$ \\
\hline Administración de Empresas & 658 & 76 \\
Contabilidad y Auditoría & 645 & 74 \\
Mercadotecnia & 425 & 49 \\
Finanzas & 652 & 75 \\
Gestión de Transporte & 571 & 66 \\
TOTAL & $\mathbf{2 . 9 5 1}$ & $\mathbf{3 4 0}$ \\
\hline
\end{tabular}

Fuente: Elaboración a partir de datos obtenidos de secretaría por cada carrera, 2020

Bernal (2006) indica que la muestra "Es la parte de la población que se selecciona, de la cual realmente se obtiene la información para el desarrollo del estudio y sobre la cual se efectuarán la medición y la observación de las variables objeto de estudio". (p. 165). Para el efecto el cálculo de la muestra fue basado en la fórmula de Vara (2012, p. 227), la misma que permitió calcular la muestra respectiva:

$$
\begin{gathered}
n=\frac{Z^{2} p \cdot q \cdot N}{e^{2}(N-1)+Z^{2} p \cdot q} \\
\mathrm{n}=\frac{\left(\left((\mathbf{1}, \mathbf{9 6})^{2} * 0,5 * 0,5\right)\right) *(2951)}{\left((2951-1) *(0,05)^{2}\right)+(1,96)^{2} * 0.5 * 0.5} \\
\mathrm{n}=340 \text { alumnos }
\end{gathered}
$$

En donde:

$\mathrm{n}=\quad$ Tamaño de la muestra definida

$\mathrm{N}=\quad$ Población considerada (2951 alumnos)

$\mathrm{Z}=\quad$ Nivel de confianza manejado $(95 \%, 1,96)$

$\mathrm{e}=\quad$ Margen de error considerado (5\%)

$\mathrm{p}=\quad$ Probabilidad de ocurrencia de un evento (50\%)

$\mathrm{q}=\quad$ Probabilidad de no ocurrencia de un evento (50\%) 
El principal instrumento que se utilizó para recopilar la información primaria es una encuesta estructurada, aplicada a 340 alumnos matriculados de la Facultad de Administración de Empresas, usando para el efecto el método el muestreo probabilístico estratificado proporcional. Para la colección de datos se utilizó la escala de Likert (1932), que de acuerdo con (Bertram, 2008) y citado por Matas (2018), es "Un instrumento psicométrico donde el encuestado debe indicar si está de acuerdo o desacuerdo sobre una afirmación, ítem o reactivo, lo cual se realiza a través de una escala ordenada y unidimensional" (p. 39).

La encuesta aplicada permitió la clasificación, el análisis y la interpretación de la información, posteriormente el programa estadístico Statistical Packge for Social Sciences (SPSS), como herramienta informática ayudo en la tabulación y procesamiento de los datos, presentados en tablas y figuras, con los analisis e interpretaciones.

\section{Rasgos generales de los alumnos encuestados}

RESULTADOS

Tabla 2. Caracterización general de los alumnos encuestados

\begin{tabular}{cl}
\hline Características analizadas & \multicolumn{1}{c}{ Hallazgos encontrados } \\
\hline Género & $\begin{array}{l}\text { Se puede observar que el 52\% pertenece al género masculino y } \\
\text { el } 48 \% \text { al sexo femenino. }\end{array}$ \\
& El 79\% de los alumnos encuestados se encuentran entre los 20 y \\
& 25 años de edad, apenas un 15\% se sitúa entre los 17 y 19 años. \\
& El $6 \%$ corresponde a estudiantes mayores a los 25 años. \\
& El $81 \%$ de los encuestados se encuentran en el sector urbano y \\
& el $19 \%$ realizan sus tareas estudiantes de forma virtual en el \\
& sector rural. \\
\hline
\end{tabular}

Fuente: Encuestas aplicadas a estudiantes FADE-ESPOCH, 2020

\section{Análisis del comportamiento de la educación virtual en época de pandemia}

\section{Nivel de preparación para iniciar estudiar en la época de la pandemia}

La pandemia por efecto de la enfermedad del coronavirus o Covit-19, tomó por sorpresa a toda la población del mundo, sin embargo, era importante para esta investigación consultar a los estudiantes en que aspectos estuvieron o no preparados para afrontar esta crisis. Los resultados registrados reflejan que en los aspectos tales como educativos (55\%), tecnológicos (52\%), económico e informático con el $48 \%$, el conjunto de los estudiantes estuvieron preparados, en cuanto a temas de investigación el $45 \%$ estuvo parcialmente preparados. (Tabla 3 ) 
Tabla 3. Nivel de preparación para iniciar a estudiar en época de pandemia (\%)

\begin{tabular}{lcccccc}
\hline \multicolumn{1}{c}{ Aspectos } & $\begin{array}{c}\text { Sin } \\
\text { preparación }\end{array}$ & $\begin{array}{c}\text { Poco } \\
\text { preparado }\end{array}$ & $\begin{array}{c}\text { Parcialmente } \\
\text { preparado }\end{array}$ & Preparado & $\begin{array}{c}\text { Muy } \\
\text { preparado }\end{array}$ & Total \\
\hline Tecnológico & 3,03 & 12,12 & 27,27 & 51,52 & 6,06 & 100,00 \\
Emocional & 0,00 & 24,24 & 30,30 & 39,39 & 6,06 & 100,00 \\
Económico & 0,00 & 33,33 & 18,18 & 48,48 & 0,00 & 100,00 \\
Informático & 6,06 & 21,21 & 21,21 & 48,48 & 3,03 & 100,00 \\
Metodológico & 6,06 & 21,21 & 33,33 & 36,36 & 3,03 & 100,00 \\
Educativo & 0,00 & 12,12 & 27,27 & 54,55 & 6,06 & 100,00 \\
Social & 3,03 & 15,15 & 33,33 & 42,42 & 6,06 & 100,00 \\
Investigativo & 3,03 & 9,09 & 45,45 & 39,39 & 3,03 & 100,00 \\
Colaborativo & 3,03 & 9,09 & 36,36 & 48,48 & 3,03 & 100,00 \\
\hline
\end{tabular}

Fuente: Encuestas aplicadas a estudiantes FADE-ESPOCH, 2020

\section{Materiales educativos utilizados en el proceso de enseñanza aprendizaje durante} la pandemia

Los materiales educativos sean físicos o digitales constituyen herramientas importantes en el proceso de enseñanza y aprendizaje, permiten a través de su consulta determinar y generar el conocimiento para generar nuevos conceptos y fortalecer la preparación académica de los educandos. Así, se puede visualizar que el $40 \%$ de los alumnos afirma que en este periodo se utilizaron libros digitales, seguido de videos (21\%) y páginas web (15\%). En menos proporción se utilizan libros impresos y revistas científicas 3\% y 9\% respectivamente. (Gráfico 1)

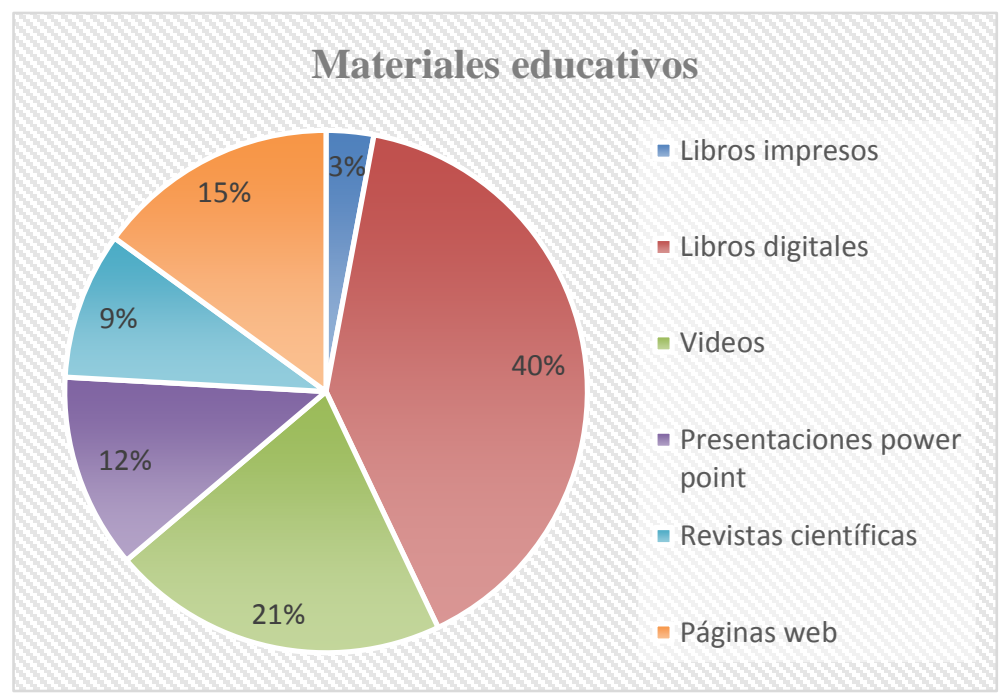

Gráfico 1. Materiales educativos utilizados en el proceso de enseñanza aprendizaje. (Fuente: Encuestas aplicadas a estudiantes FADE-ESPOCH, 2020). 


\section{Recursos tecnológicos utilizados en el proceso de enseñanza aprendizaje}

Los recursos tecnológicos representan medios o bienes educativos tangibles que los alumnos los utilizan, que para cumplir sus objetivos y propósitos hacen uso de la tecnología. Las respuestas dadas por los estudiantes indican que el $49 \%$ de los alumnos han utilizado computadores portátiles, seguido de computadores de escritorio (27\%). Los teléfonos móviles (15\%) han sido los menos utilizados. (Gráfico 2).

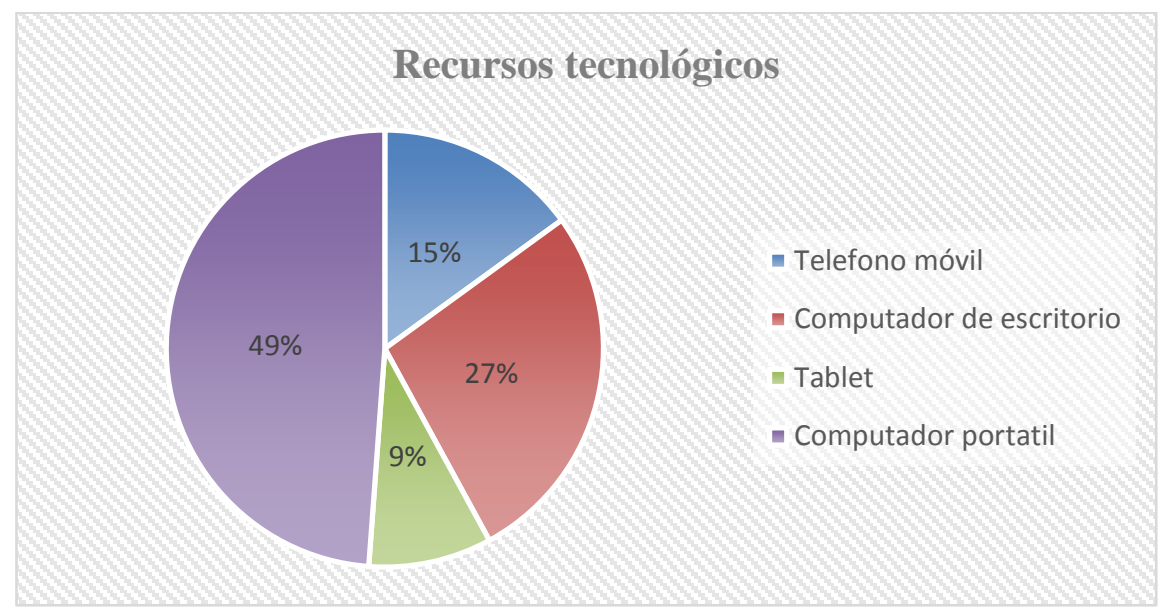

Gráfico 2. Recursos tecnológicos utilizados en el proceso de enseñanza aprendizaje durante la pandemia. (Fuente: Encuestas aplicadas a estudiantes FADE-ESPOCH, 2020).

\section{Calificación del proceso de aprendizaje durante la pandemia}

Los recursos tecnológicos son los medios o productos educativos que los alumnos hacen uso a través de la tecnología y que permiten cumplir con los propósitos educativos, en este contexto el tiempo que den uso a los equipos depende de la cantidad de trabajos y tareas que dispongan, por ello se desprende que el $82 \%$ considera que fue bueno el tiempo de uso de los recursos tecnológicos, al igual que el 15\% afirmó que el tiempo de uso fue regular. (Gráfico 3).

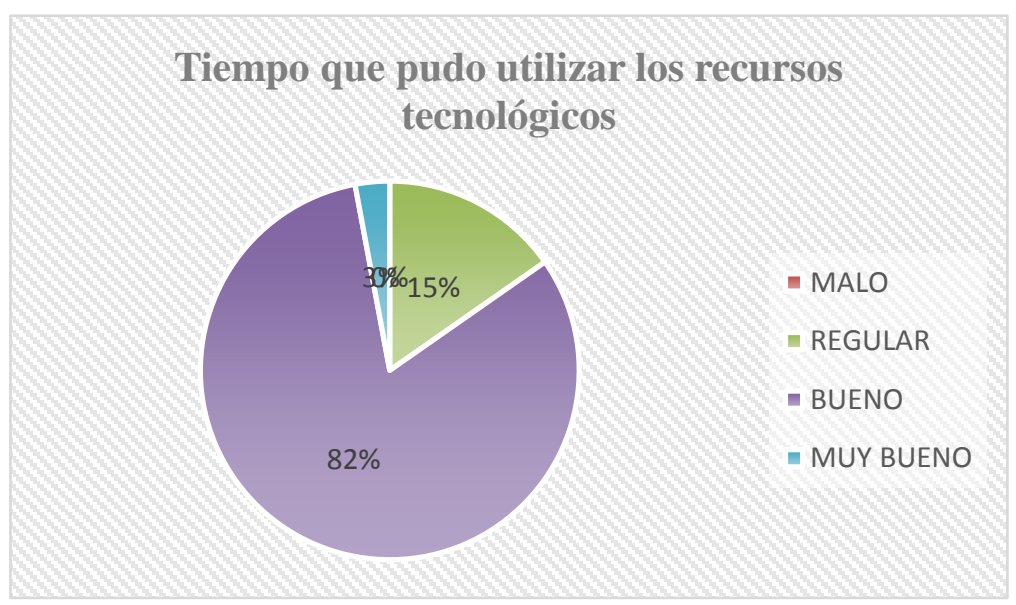

Gráfico 3. Calificación de la cantidad de tiempo de uso de los recursos tecnológicos. Fuente: Encuestas aplicadas a estudiantes FADE-ESPOCH, 2020 
Como complemento al uso de los equipos y recursos tecnológicos, es importante disponer de un servicio de internet sea público o privado en condiciones óptimas y que permitan desarrollar las actividades académicas y de enseñanza aprendizaje, así el 51\% manifestó que el servicio de internet fue bueno y el 37\% regular. (Gráfico 4).

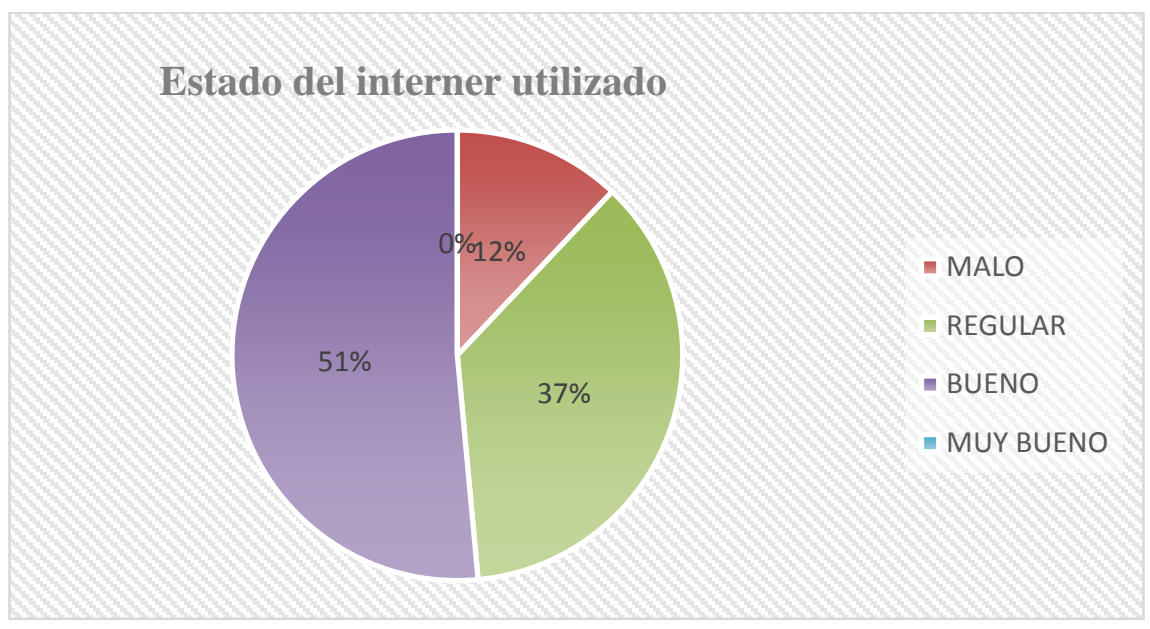

Gráfico 4. Calificación del uso del internet. (Fuente: Encuestas aplicadas a estudiantes FADE-ESPOCH, 2020).

Los equipos informáticos utilizados por los estudiantes de carreras empresariales, son computadores portátiles (Laptop), y de escritorio en su mayoría, los datos de la encuesta arrojan los siguientes resultados: El 49\% y 39\% respectivamente manifiestan que el estado de los equipos que están utilizando en el proceso de enseñanza y aprendizaje, fue bueno y regular. Apenas un 6\% afirmaron que el estado de los equipos fue muy bueno. (Gráfico 5).

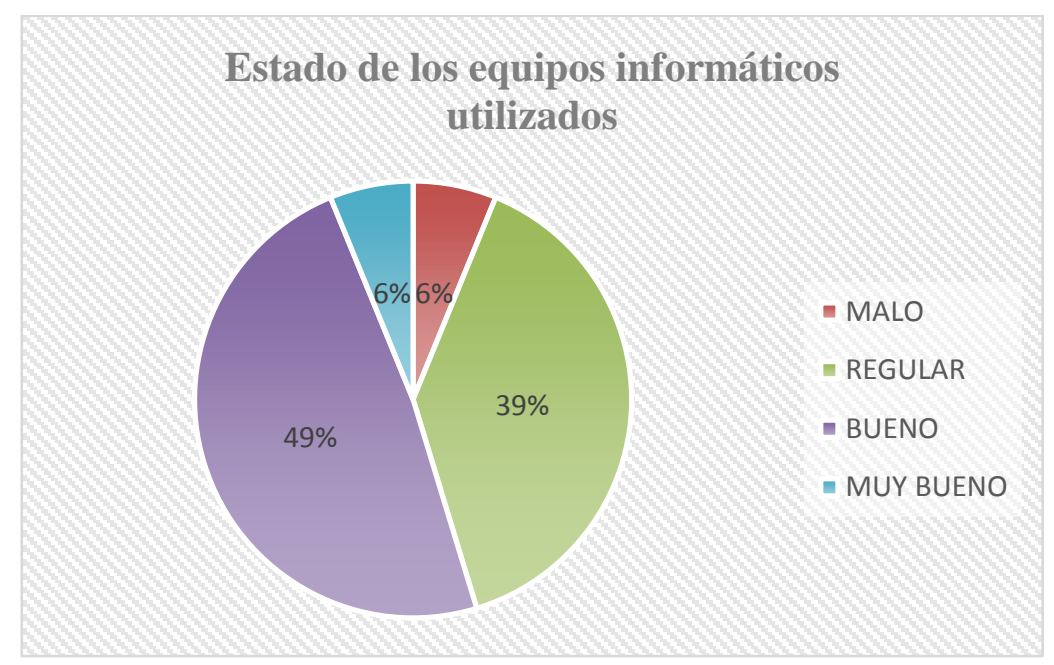

Gráfico 5. Calificación del estado de los equipos informáticos utilizados. Fuente: Encuestas aplicadas a estudiantes FADE-ESPOCH, 2020. 
Actividades académicas realizadas durante el semestre correspondiente a la pandemia

Durante el periodo académico dentro de la pandemia los docentes de educación superior han aplicado un conjunto de actividades académicas para cumplir con el proceso de formación (Enseñanzaaprendizaje) en favor de sus educandos, las clases virtuales han sido las que más se destacan con el 94\%, seguido en importancia la investigación bibliográfica (85\%). Debido a las características del sistema de educación actual los trabajos grupales, individuales y la investigación formativa registran una participación importante con el $82 \%$ respectivamente; en tanto que comparando las actividades sincrónicas y asincrónicas se han utilizado las segundas (Asincrónicas) con el 64\%. (Tabla 4).

Tabla 4. Asociación del tipo de actividades académicas realizadas durante el periodo académico correspondiente a la pandemia.

\begin{tabular}{lc}
\hline \multicolumn{1}{c}{ ACTIVIDADES ACADÉMICAS } & $\begin{array}{c}\text { \% REALIZADAS DURANTE LA } \\
\text { PANDEMIA }\end{array}$ \\
\hline CLASES VIRTUALES & 93,94 \\
TUTORÍAS DOCENTES & 78,79 \\
TRABAJOS GRUPALES & 81,82 \\
TRABAJOS INDIVIDUALES & 81,82 \\
JUEGOS EDUCATIVOS & 33,33 \\
PRACTICAS VIRTUALES & 42,42 \\
ACTIVIDADES SINCRONAS (mismo tiempo) & 57,58 \\
ACTIVIDADES ASÍNCRONAS (tiempo diferido) & 63,64 \\
INVESTIGACIÓN FORMATIVA & 81,82 \\
INVESTIGACIÓN BIBLIOGRÁFICA & 84,85 \\
\hline
\end{tabular}

Fuente: Encuestas aplicadas a estudiantes FADE-ESPOCH, 2020

Relación entre la actividad académica y el software utilizado durante la pandemia

Dentro del proceso de enseñanza aprendizaje en el sistema universitario, es importante desarrollar un conjunto de actividades académicas, las mismas que vengan acompañadas del uso de diferentes softwares que se encuentran con el uso del internet, a través de actividades sincrónicas y asincrónicas, así como constantes en las redes sociales de mayor uso. Así las clases virtuales (94\%), Tutorías docentes (94\%), Prácticas virtuales (64\%) y trabajos grupales (61\%), han sido las actividades académicas más desarrolladas en el sistema teams. Mientras que en el sistema zoom se ha desarrollado juegos educativos con un $27 \%$ de participación. Dentro de las redes sociales el Facebook ha sido la de menor utilización en actividades académicas. (Tabla 5). 
Tabla 5. Relación entre las actividades académicas y el software utilizado durante a la pandemia

\begin{tabular}{lccccccc}
\hline \multicolumn{1}{c}{$\begin{array}{c}\text { Actividades } \\
\text { académicas }\end{array}$} & Moodle & Teams & Zoom & Whatsapp & Facebook & $\begin{array}{c}\text { Web } \\
\text { institucional }\end{array}$ & Total \\
\hline Clases virtuales & 0,00 & 93,94 & 6,06 & 0,00 & 0,00 & 0,00 & 100,00 \\
Tutorías docentes & 0,00 & 93,94 & 6,06 & 0,00 & 0,00 & 0,00 & 100,00 \\
Trabajos grupales & 3,03 & 60,61 & 6,06 & 18,18 & 0,00 & 12,12 & 100,00 \\
Trabajos & 6,06 & 39,39 & 6,06 & 9,09 & 0,00 & 39,39 & 100,00 \\
individuales & 0,00 & 48,48 & 27,27 & 18,18 & 3,03 & 3,03 & 100,00 \\
Juegos educativos & 3,03 & 63,64 & 9,09 & 6,06 & 0,00 & 18,18 & 100,00 \\
\hline Practicas virtuales & & & & & & &
\end{tabular}

Fuente: Encuestas aplicadas a estudiantes FADE-ESPOCH, 2020

\section{Calificación de la investigación antes y durante la pandemia}

En El Sistema De Educación Superior La Investigación Es Uno De Los 4 Pilares De Desarrollo Institucional, Además De La Vinculación, Gestión Y Docencia; Por Ello Es Importante Medir La Calificación Que Los Estudiantes Dieron A La Investigación Antes Y Durante La Pandemia, Antes De La Pandemia El 36\% Afirmaron Que La Investigación Fue Buena Mientras Que Los Que Calificaron Muy Bueno Representan El 58\%. Durante La Pandemia Quienes Calificaron De Bueno Subió Al 52\% Y Quienes Indicaron Que Es Muy Bueno Disminuyó Al 39\%. (Tabla 6).

Tabla 6. Calificación de la investigación antes y durante la pandemia

\begin{tabular}{lccrr}
\hline \multicolumn{1}{r}{ CALIFICACIÓN } & Antes de la Pandemia & \% & Durante la pandemia & \multicolumn{1}{c}{} \\
\hline Pésimo & 0 & 0,00 & 0 & 0,00 \\
Malo & 0 & 0,00 & 12 & 3,53 \\
Regular & 20 & 5,88 & 19 & 5,59 \\
Bueno & 123 & 36,18 & 178 & 52,35 \\
Muy Bueno & 197 & 57,94 & 131 & 38,53 \\
\hline \multicolumn{1}{c}{ TOTAL } & $\mathbf{3 4 0}$ & $\mathbf{1 0 0 , 0 0}$ & $\mathbf{3 4 0}$ & $\mathbf{1 0 0 , 0 0}$ \\
\hline
\end{tabular}

Fuente: Encuestas aplicadas a estudiantes FADE-ESPOCH, 2020

\section{Calificación de la enseñanza de los profesores antes y durante la pandemia}

El sistema de educación superior promueve que los educandos reciban una educación de calidad de parte de sus docentes, es así que se evalúo este factor antes y durante la pandemia, obteniéndose los siguientes resultados: Antes de la pandemia los alumnos manifestaron en un $63 \%$ que la enseñanza fue muy buena, mientras que durante la pandemia disminuyó al 41\%. (Tabla 7). 
Tabla 7. Calificación de la enseñanza de los profesores antes y durante la pandemia

\begin{tabular}{lccrrr}
\hline \multicolumn{1}{c}{ CALIFICACIÓN } & Antes de la Pandemia & \% & Durante la pandemia & \% \\
\hline Pésimo & 0 & 0,00 & 0 & 0,00 \\
Malo & 0 & 0,00 & 34 & 10,00 \\
Regular & 15 & 4,41 & 52 & 15,29 \\
Bueno & 112 & 32,94 & 115 & 33,82 \\
Muy Bueno & 213 & 62,65 & 139 & 40,88 \\
\hline TOTAL & $\mathbf{3 4 0}$ & $\mathbf{1 0 0 , 0 0}$ & $\mathbf{3 4 0}$ & $\mathbf{1 0 0 , 0 0}$ & \\
\hline
\end{tabular}

Fuente: Encuestas aplicadas a estudiantes FADE-ESPOCH, 2020

\section{Calificación del aprendizaje de los alumnos antes y durante la pandemia}

Complementando al sistema de enseñanza que los docentes ofrecen a sus estudiantes, fue relevante medir el aprendizaje recibido por parte de los alumnos, así los educandos han manifestado como se muestra en la Tabla 8 que su aprendizaje ha disminuido durante la pandemia (Muy bueno y bueno $80 \%$ ), frente a lo sucedido antes de la pandemia ( $97 \%$ entre las dos calificaciones).

Tabla 8. Calificación del aprendizaje antes y durante la pandemia

\begin{tabular}{lccrrr}
\hline \multicolumn{1}{r}{ CALIFICACIÓN } & Antes de la Pandemia & \% & Durante la pandemia & \multicolumn{1}{c}{} \\
\hline Pésimo & 0 & 0,00 & 6 & 1,76 \\
Malo & 0 & 0,00 & 37 & 10,88 \\
Regular & 9 & 2,65 & 23 & 6,76 \\
Bueno & 121 & 35,59 & 106 & 31,18 \\
Muy Bueno & 210 & 61,76 & 168 & 49,41 \\
\hline \multicolumn{1}{c}{ TOTAL } & $\mathbf{3 4 0}$ & $\mathbf{1 0 0 , 0 0}$ & $\mathbf{3 4 0 , 0 0}$ & $\mathbf{1 0 0 , 0 0}$ \\
\hline
\end{tabular}

Fuente: Encuestas aplicadas a estudiantes FADE-ESPOCH, 2020

\section{Calificación de la evaluación antes y durante la pandemia}

Tabla 9. Calificación de la evaluación antes y durante la pandemia

\begin{tabular}{lrrrrrc}
\hline Cuestionamientos/Calificación & Pésimo & Malo & Regular & Bueno & Muy bueno & Total \\
\hline Antes de la cuarentena & 0,00 & 0,00 & 12,12 & 48,48 & 39,39 & 100,00 \\
Durante la cuarentena & 0,00 & 0,00 & 21,21 & 63,64 & 15,15 & 100,00 \\
\hline
\end{tabular}

Fuente: Encuestas aplicadas a estudiantes FADE-ESPOCH, 2020 
Producto del proceso de enseñanza y aprendizaje de los docentes hacia sus estudiantes, fue importante realizar una evaluación de conocimientos durante el semestre, así se pudo verificar que antes de la pandemia el 39\% indicaba que la evaluación era muy buena, así durante la pandemia se afirmó que la evaluación bajó a un 15\%.

\section{Para el próximo periodo académico 2020-2021, cuál sería el nivel de acuerdo con la modalidad de estudios}

Tabla 10. Modalidad de estudio a aplicarse en el próximo periodo académico

\begin{tabular}{ccccccc}
\hline Modalidad & $\begin{array}{c}\text { Totalmente } \\
\text { en } \\
\text { desacuerdo }\end{array}$ & $\begin{array}{c}\text { En } \\
\text { desacuerdo }\end{array}$ & $\begin{array}{c}\text { Ni de } \\
\text { acuerdo } \\
\text { ni en } \\
\text { desacuerdo }\end{array}$ & $\begin{array}{c}\text { De } \\
\text { acuerdo }\end{array}$ & $\begin{array}{c}\text { Totalmente } \\
\text { de acuerdo }\end{array}$ & Total \\
\hline $\begin{array}{c}\text { Virtual totalmente } \\
\text { sincrónico (mismo } \\
\text { tiempo) (\%) }\end{array}$ & 0,00 & 34,02 & 17,89 & 39,00 & 9,09 & 100,00 \\
$\begin{array}{c}\text { Virtual totalmente } \\
\text { asincrónico (Tiempo } \\
\text { diferido) \% }\end{array}$ & 0,00 & 20,94 & 35,99 & 40,12 & 2,95 & 100,00 \\
$\begin{array}{c}\text { Virtual sincrónico y } \\
\text { asincrónico (\%) }\end{array}$ & 0,00 & 20,94 & 35,99 & 37,17 & 5,90 & 100,00 \\
$\begin{array}{c}\text { Presencial (\%) } \\
\begin{array}{c}\text { Presencial y virtual } \\
\text { (\%) }\end{array}\end{array} \quad 10,00$ & 15,00 & 12,06 & 27,06 & 35,88 & 100,00 \\
\hline
\end{tabular}

Fuente: Encuestas aplicadas a estudiantes FADE-ESPOCH, 2020

La pandemia de alguna forma ha afectado al sector estudiantil y docente en el sistema de educación superior, tanto en lo económico, tecnológico, afectivo, y en su nivel aprendizaje educativo, por ello los estudiantes de educación superior han manifestado que para los próximos semestres de estudio se realice bajo la modalidad presencial el $63 \%$, y en forma combinada entre presencial y virtual el $60 \%$.

\section{Discusión}

Los resultados encontrados en la investigación permitieron observar que existe mayor cantidad de población estudiantil del género masculino en carreras empresariales, mientras la edad para estudiar este tipo de especialidades está entre los 19 y 25 años, siendo edad normal para cursar estudios universitarios desde el inicio hasta la obtención de su título académico. En relación al lugar de precedencia más de las dos terceras partes pertenecen al sector urbano que realizan clases bajo el sistema virtual, por lo que se considera que las condiciones tecnológicas, educativas y económicas son mejores al estar en el área urbana que en el área rural.

En la época de pandemia por efecto del coronavirus o Covit-19, en el Ecuador y el mundo la mayoría de instituciones de educación superior tanto públicas como 
privadas realizan sus actividades académicas de forma irregular, por efecto de la cuarentena o confinamiento, esto obviamente ha generado preocupación en todos los aspectos, y especialmente en el sistema educativo, en relación a los procesos de enseñanza y aprendizaje. Por ello, y como lo manifiesta Moreno (2020), La situación actual que atraviesa la humanidad es bastante preocupante y lleva a que los habitantes de un planeta que se encuentra en un proceso de devastación reflexionen e implementen cambios en su estilo de vida (...) (p. 24).

La pandemia por el coronavirus tomó de manera imprevista a toda la humanidad debido al aparecimiento de la enfermedad, por ello se tuvo que recurrir a la educación bajo la modalidad virtual, como lo dice Alcívar (2021), "Pasamos a entornos digitales de una manera abrupta. La educación online como está concebida tiene una metodología diferente, responde a un público distinto, tiene ciertas características (...)” (p. 10). Sin embargo, a esta circunstancia los estudiantes demuestran en gran medida una buena preparación para enfrentar la crisis de la pandemia, en aspectos educativos, tecnológicos, económicos e informáticos.

Debido al sistema de enseñanza aprendizaje durante la pandemia, la mayoría de docentes han utilizado los libros digitales, videos y páginas web para desarrollar su proceso educativo en favor de sus alumnos. Si este estudio se compara con similares realizados en otros países en época de pandemia, en relación a los recursos tecnológicos manifiestan que: "Con relación a la implementación de recursos pedagógicos digitales se pudo observar que, al ser tan precipitada esta situación de confinamiento social, los docentes se volcaron a la digitalización de material didáctico preexistente y la elaboración de guías de estudio" (Expósito y Marsollier, 2020, p. 18).

Por tanto, se evidenció, el uso de diferentes recursos tecnológicos de manera virtual debido a la situación de la modalidad de estudio, que a decir del conjunto de estudiantes en la realización de actividades y tareas el uso de los mismos fue bueno el uso afirmándose un $82 \%$ de los alumnos, usando para el efecto computadores portátiles en su gran mayoría. En este contexto el uso del servicio de internet es más que indispensable, sin embargo, de existir empresas públicas y privadas que ofrecen este tipo de servicios, la mitad de los alumnos afirmaron que para su preparación académica el servicio fue bueno; adicional que el estado de los equipos en un $50 \%$ indicaron que se encontraban en buen estado para uso de actividades académicas.

Sobre estas temáticas, por tanto, es necesario tener referencias con otro tipo de investigaciones, por ello como lo manifiesta en su estudio Beltrán y Vanegas (2020):

(...) hay alrededor de un $20 \%$ de familias que no disponen de ordenador o de conexión a internet. Esta cuestión es imprescindible para poder seguir la enseñanza no presencial. Aparte, aún las familias que tienen, algunas necesitan el ordenador por el teletrabajo de la madre y/o el padre, o bien, algunos progenitores han de desplazarse al lugar de trabajo (...). Esto dificulta el aprendizaje a este alumnado. ( $p$ 101).

En el caso de los estudiantes de carreras empresariales de la Escuela Superior Politécnica de Chimborazo (ESPOCH), sin embargo, de disponer de recursos tecnológicos, así como del servicio de internet público o privado en condiciones aceptables para su 
proceso de formación académica y educativa, no lo tienen en las condiciones como las que se requieren para obtener una educación superior de excelencia y calidad, en donde los procesos educativos beneficien al sector estudiantil.

Al ser una etapa de estudios atípico en el mundo, el único mecanismo que queda a las autoridades educativas es hacer uso del internet para las clases virtuales u online, en pos de cumplir con el proceso educativo, en este esquema los docentes en favor de sus alumnos han enviado trabajos grupales e individuales a sus alumnos, y bajo la modalidad asincrónica se ha utilizado en mayor porcentaje, es decir en modalidad de estudio no en línea. Para el efecto se ha utilizado el sistema teams en gran medida seguido del zoom para el dictado de clases en línea.

La ley de educación superior en el Ecuador promueve que los estudiantes a través del dictado de clases de sus docentes obtengan una educación de calidad, es por ello que los alumnos han calificado la enseñanza de sus profesores; se puede evidenciar por tanto que antes de la pandemia la enseñanza tenía una calificación de muy buena, disminuyendo durante la época de la pandemia. Se puede decir, por tanto, que:

El ejercicio docente, es una labor que implica formar un ser humano en lo cognitivo y la práctica, el docente frente a la sociedad y al Estado se hace responsable de la conducción del aprendizaje de un ser humano en sus distintas etapas para continuar el proceso formativo (Borges, 2019, p. 47).

Al proceso de enseñanza de los docentes es importante complementar con el aprendizaje recibido o captado por todos sus estudiantes, evidenciándose que debido a la modalidad de clases dictadas $\mathrm{y}$ al proceso recibido, entre antes de la pandemia y durante la misma el aprendizaje de los alumnos ha disminuido de forma sustancial perjudicando obviamente a los educandos. Es importante de la misma manera reflexionar por ello, que: "Resulta esencial entonces, prestar atención al rol que juegan tanto docente como discente, para que sea efectivo el proceso de enseñanza aprendizaje" (Chacón, 2019, p. 59).

Como complemento a los procesos de formación educativa, se conoce por la Ley de Educación Superior en el Ecuador, los 4 pilares de donde se sustenta la educación superior son: la docencia, gestión, vinculación e investigación; notándose que luego de los procesos de docencia se realizan evaluaciones de los docentes a los estudiantes, advirtiéndose que este aspecto ha disminuido en su parte de exigencia; al igual que los procesos de investigación, por cuanto no se dispone del tiempo y los recursos académicos y tecnológicos para su ejecución.

El confinamiento por efecto de la pandemia del Covit-19, ha afectado de manera sustancial a todas las actividades económicas, sociales, culturales, productivas, y más aún al sistema educativo, por tanto se puede notar una gran preocupación en los estudiantes de carreras empresariales de la Escuela Superior Politécnica de Chimborazo, en que la modalidad de clases se cambie, es decir se vuelva a las clases presenciales, es por ello que los estudiantes de educación superior han indicado que para los próximos semestres de estudio se realice bajo la modalidad presencial o en forma combinada entre presencial y virtual, notándose entre las 2 terceras partes de los alumnos.

Para cerrar en este contexto, y a manera de reflexión final Menéndez (2012), citado por Moreno (2020) indica que las directrices acerca de la virtualidad, suponen un reto para el 
quehacer docente, encontrando de esta manera un desafío para las instituciones, docentes y sus estudiantes se encuentran familiarizados con un solo modelo educativo, el modelo tradicional de enseñanza-aprendizaje a través de clases magistrales, pues migrar desde este punto al modelo virtual genera sentimientos de angustia, desconfianza e incertidumbre para ambas partes". (p. 17).

\section{CONCLUSIONES}

Debido al confinamiento por efecto de la pandemia del coronavirus o Covit-19, enfermedad que ha aparecido no solo en el Ecuador sino a nivel mundial, las diferentes actividades de la sociedad, y especialmente las del sistema de educación superior, han sido afectadas $y$ han obligado a cambiar las estrategias de enseñanza y aprendizaje en la formación educativa de los educandos.

Estos cambios han trasladado de la educación tradicional presencial, mediante clases magistrales en donde los estudiantes comparten de manera directa con sus docentes; a la educación virtual u online.

El sistema virtual o en línea de educación en el nivel superior, posee ciertas características o particularidades, que lo hacen muy diferente de la educación presencial, en donde los docentes pueden compartir con sus estudiantes, adicional realizar un conjunto de actividades que pueden ayudar a fortalecer la formación académica de los alumnos, mejorando inclusive la relación afectiva entre sus compañeros de aula y sus diversos docentes, se pueden desarrollar incluso otro tipo de competencias y habilidades que no se los puede hacer bajo la modalidad virtual.

La modalidad virtual utilizada para el sistema educativo, es parte de la nueva normalidad que viven los países en el mundo, en donde las personas no solo deben preocuparse por la educación, sino por mantener su trabajo o actividad laboral sea pública o privada, mantener niveles de salud aceptables para desarrollar otras actividades como la educativa. Por ello se puede afirmar que la educación virtual no solo ha permitido meditar y analizar que ha afectado en los sistemas de salud de los estudiantes y docentes, así como en los aspectos específicos de la educación.

Los principales resultados revelan que los niveles de enseñanza de los docentes, así como el aprendizaje de los estudiantes, a través de las diferentes estrategias educativas implementadas por sus docentes comparando antes y durante la pandemia han disminuido de manera considerable, así como los procesos de evaluación e investigación, en este caso no existen las condiciones y los recursos necesarios e indispensables para desarrollar procesos investigativos de manera eficiente.

Por ello, es importante que los docentes adopten posturas de mejorar su proceso de enseñanza, buscando estrategias de formación más eficientes, acordes a cada uno de sus alumnos y en función a la realidad de la modalidad de educación; de la misma forma los estudiantes deben tener mayor compromiso y autopreparación, demostrando mayor responsabilidad en su nivel de aprendizaje. Por ello, tanto docentes como estudiantes debe ser más creativos e innovadores que ayuden a mejorar su formación académica.

\section{REFERENCIAS}

Bernal, C. (2006). Metodología de la Investigación. Para administración, economía, humanidades y ciencias sociales. México: Pearson Educación

Beltrán, J., y Vanegas, M. (2020). Educar en época de confinamiento: La tarea de renovar un mundo común. Revista de Sociología de la Educación. Vol. 13, núm. 2. Especial Covid-19, pp. 92-104 
Borges, M. E. (2019). La revalorización docente: realidades y paradigmas. Revista Revencyt del Centro de Investigación y Estudios Gerenciales (CIEG), número 37, mayo-junio 2019, pp. 47-59

Bravo-García, E., y Magis-Rodríguez, C. (2020). La respuesta mundial a la epidemia del COVID-19: los primeros tres meses. Boletín sobre COVID-19 Salud Pública y Epidemiología, Vol. 1 número 1, pp. 3-8. http://dsp.facmed.unam.mx/wpcontent/uploads/2013/12/COVID-19-No.103-La-respuesta-mundial-a-la-epidemiadel-COVID-19-los-primeros-tres-meses.pdf

Ceballos, A., Arévalo, B., y Giraldo, J. (2012). Plan de Marketing para incrementar el número de estudiantes en el Centro Educativo la Sabiduría de la Ciudad de Barranquilla. Revista Escenarios, Vol. 10, No. 1, pp. 29-39. Recuperado de: file:///C:/Users/Usuario/Downloads/Dialn et-

PlanDeMarketingParaIncrementarElNumer oDeEstudiante-4495604.pdf

Chacón, R. (2019). Desarrollo de habilidades metacognitivas de estudiantes durante el desarrollo de la asignatura seminario de Investigación I. Revista Revencyt del Centro de Investigación y Estudios Gerenciales (CIEG), número 38, julio-agosto 2019, pp. 58- 73

Expósito, C., y Marsollier, R. (2020). Virtualidad y educación en tiempos de COVID-19. Un estudio empírico en Argentina. Revista Educación y Humanismo, 22(39), pp.1-22. DOI:

https://doi.org/10.17081/eduhum.22.39.4 214file://C:/Users/GlobalOffice/Downloa ds/4214-

Texto\%20del\%20art\%C3\%ADculo-148392-10-20200831.pdf

Flores, J. (2011). Construyendo la tesis universitaria. Guía Didáctica. Lima: Garden Graf S.R.L

Gutiérrez, L. (2016). Deliberación entorno a la Educación Virtual. Interconectando Saberes, vol. (1), pp. 77-89.

http://is.uv.mx/index.php/IS/article/view/ 1112
Hurtado, F. (2020). La Educación en tiempos de pandemia: Los desafíos de la escuela del siglo XXI, Revista Revencyt del Centro de Investigación y Estudios Gerenciales (CIEG), número 44, julio-agosto 2020, pp. 176-187. ISSN: 2244-8330

BID. Inter-american Development Bank. (2020). La educación en tiempos del coronavirus. Los sistemas educativos en América Latina y el Caribe ante COVIT-19. Disponible en: https://publications.iadb.org/publications/ spanish/document/La-educacion-entiempos-del-coronavirus-Los-sistemaseducativos-de-America-Latina-y-el-Caribeante-COVID-19.pdf

Marciniak, R., y Gairín-Sallán, J. (2018). Dimensiones de evaluación de calidad de educación virtual: revisión de modelos referentes. RIED. Revista Iberoamericana de Educación a Distancia, 21(1), pp. 217-238. Disponible https://doi.org/10.5944/ried.21.1.16182

Matas, A. (2018). Diseño del formato de escalas tipo Likert: Un estado de la cuestión. Revista Electronica de Investigacion Educativa, Vol 20, Núm 1, pp. 38-47

Moreno, S. (2020). La innovación educativa en los tiempos del coronavirus. Revista Salutem Scientia Spiritus, 6 (1), pp. 14-26. Recuperado de: https://pesquisa.bvsalud.org/portal/resour ce/pt/biblio-1087909

NACIONES UNIDAS (2020). Informe de políticas: La educación durante la COVID-19 y después de ella AGOSTO DE 2020. https://www.un.org/sites/un2.un.org/files /policy_brief_-_education_during_covid19_and_beyond_spanish.pdf

ORGANIZACIÓN MUNDIAL DE LA SALUD -OMS(2020). Recuperado de: https://www.who.int/es/healthtopics/coronavirus

Reynoso, M., Pernas, I., y Perazzo, C. (2017). Marketing Educativo en CONALEP Monterrey I: Una estrategia heterogénea y armonizada. Revista Cubana Educación Superior. 2. pp. 102-112. Recuperado de: 
http://scielo.sld.cu/pdf/rces/v36n2/rces10 217.pdf

UNESCO (Organización de las Naciones Unidas para la Educación, la Ciencia y la Cultura) (2020), "Interrupción educativa y respuesta al COVID-19" [en línea] https://es.unesco.org/Covit19/educationresponse

Valladares, C., y Poma, V. (2015). La percepción en los estudiantes del tercer año de bachillerato de los colegios fiscales del sector urbano del cantón Riobamba, sobre las pruebas de admisión que realiza la Senescyt para ingresar a las instituciones de educación superior, periodo 2014-2015. Tesis de pregrado, Escuela Superior
Politécnica de Chimborazo, Facultad de Administración de Empresas, Escuela de Ingeniería en Marketing

Vara-Horna, A. (2012). Desde La Idea hasta la sustentación: Siete pasos para una tesis exitosa. Un método efectivo para las ciencias empresariales. Instituto de Investigación de la Facultad de Ciencias Administrativas y Recursos Humanos. Universidad de San Martín de Porres. Lima

Velásquez, B. (2020). La educación virtual en tiempos de Covid-19. Universidad de San Carlos de Guatemala, 3(1), pp. 19-25. DOI: https://doi.org/10.46734/revcientifica. v2i1.8 